\title{
Neural Dedifferentiation across the Lifespan in the Motor and Somatosensory Systems
}

\author{
Kaitlin Cassady ${ }^{1}$, Marit F.L. Ruitenberg ${ }^{2,3}$, Patricia A. Reuter-Lorenz ${ }^{1}$, \\ Mark Tommerdahl ${ }^{4}$ and Rachael D. Seidler ${ }^{5}$
}

${ }^{1}$ Department of Psychology, University of Michigan, Ann Arbor, MI 48103, USA, ${ }^{2}$ Department of Experimental Psychology, Ghent University, Ghent 9000, Belgium, ${ }^{3}$ Department of Health, Medical and Neuropsychology, Leiden University, Leiden 2300, The Netherlands, ${ }^{4}$ Department of Biomedical Engineering, University of North Carolina, Chapel Hill, NC 27514, USA and ${ }^{5}$ Department of Applied Physiology \& Kinesiology, University of Florida, Gainesville, FL 32611, USA

Address correspondence to Rachael Seidler, Department of Applied Physiology \& Kinesiology, University of Florida, 1864 Stadium Road, Gainesville, FL 32611, USA. Email: rachaelseidler@ufl.edu

\begin{abstract}
Age-related declines in sensorimotor performance have been linked to dedifferentiation of neural representations (i.e., more widespread activity during task performance in older versus younger adults). However, it remains unclear whether changes in neural representations across the adult lifespan are related between the motor and somatosensory systems, and whether alterations in these representations are associated with age declines in motor and somatosensory performance. To investigate these issues, we collected functional magnetic resonance imaging and behavioral data while participants aged 19-76 years performed a visuomotor tapping task or received vibrotactile stimulation. Despite one finding indicative of compensatory mechanisms with older age, we generally observed that 1) older age was associated with greater activity and stronger positive connectivity within sensorimotor and LOC regions during both visuomotor and vibrotactile tasks; 2) increased activation and stronger positive connectivity were associated with worse performance; and 3) age differences in connectivity in the motor system correlated with those in the somatosensory system. Notwithstanding the difficulty of disentangling the relationships between age, brain, and behavioral measures, these results provide novel evidence for neural dedifferentiation across the adult lifespan in both motor and somatosensory systems and suggest that dedifferentiation in these two systems is related.
\end{abstract}

Key words: aging, connectivity, dedifferentiation, sensorimotor, task activity

\section{Introduction}

Normal aging is associated with both structural and functional brain changes (Damoiseaux 2017), which are thought to contribute to age-related declines in sensorimotor functioning (Seidler et al. 2010). Understanding the neural mechanisms underlying these declines is important because most activities of daily living require skilled actions that depend upon sensorimotor processes. Prior work has shown that functional brain activation is more widespread and bilateral in older relative to young adults during a variety of cognitive tasks (Cabeza 2002; Reuter-Lorenz and Lustig 2005). In addition, accumulating evidence demonstrates that older adults exhibit similar overactivation during motor tasks (Mattay et al. 2002; Heuninckx et al. 2005, 2008). Some studies have reported that increased (i.e., more widespread) activation in older adults is associated with better performance (i.e., that it is compensatory) (Cabeza 2002; Reuter-Lorenz 2002; Reuter-Lorenz and Lustig 2005). In contrast, other studies have found that increased activation during task 
engagement in older as compared to younger adults is either irrelevant for the task or associated with worse performance (i.e., dedifferentiation) (Riecker et al. 2006; Heuninckx et al. 2008; Langan et al. 2010; Park et al. 2010; Bernard and Seidler 2012). In the cognitive domain, evidence is mostly observed for the compensation account (Cabeza et al. 2018). However, in the motor domain, the pattern of findings is less consistent.

Overactivation in the motor system in older adults during motor task performance is typically evident by increased engagement of the motor cortex ipsilateral to the moving hand (Mattay et al. 2002; Heuninckx et al. 2005, 2008; Langan et al. 2010). This increased ipsilateral activity may be the result of age-related changes in inter inhibition in the motor system. Supporting this interpretation, our group has shown decreased interhemispheric inhibition in older relative to young adults using transcranial magnetic stimulation (Fling and Seidler 2012). Moreover, this measure of inhibition was associated with individual differences in asynchronous bimanual movement performance in young and older adults. Similarly, Fujiyama et al. (2012) observed that inhibitory function was reduced in older adults and that this reduction was associated with worse performance on an interlimb coordination task (Fujiyama et al. 2012). While the majority of work on this topic has focused on bimanual neural representations, fewer studies have investigated both unimanual and bimanual representations and associated behavioral performance. Given that intrahemispheric inhibition also declines with age (Peinemann et al. 2001), it stands to reason that less distinct unimanual neural representations (i.e., increased activity during task engagement in older vs. younger adults) may be an underlying factor.

It is unclear whether age-related neural dedifferentiation is specific to the motor system or whether it is also characteristic of the somatosensory system. Previous studies have demonstrated that somatosensory (i.e., vibrotactile) processing declines with age (Zhang et al. 2011). In addition, our group recently demonstrated that inter and intrahemispheric inhibition also declines with age in the somatosensory system (Ruitenberg et al. 2019). Whether this age difference in surround inhibition (or somatosensory performance in general) is associated with differences in somatosensory neural representations remains an open question. Furthermore, whether age effects in somatosensory neural representations are related to those in the motor system is also unknown.

In the present study, we evaluated both unimanual and bimanual motor and somatosensory representations in individuals ranging from 19 to 76 years old to investigate whether these representations are associated with age differences in motor and somatosensory performance. We collected functional magnetic resonance imaging (fMRI) and behavioral data while participants performed a visuomotor tapping task and a tactile task. In the visuomotor task, they moved either one digit or two digits asynchronously, for which the movements were visually cued. In the tactile task, they received vibrotactile stimulation to either one digit or two digits simultaneously.

We also performed functional connectivity MRI (fcMRI) analysis on the task-based fMRI data in order to further probe the neural mechanisms underlying age-related dedifferentiation in the motor and somatosensory systems. We employed a seed-tovoxel fCMRI approach using brain regions from the fMRI results which exhibited an association with either age or behavior as seed regions. We subsequently examined the correlation between these seed regions with the rest of the brain during task performance.

In terms of functional activation, we hypothesized that 1) increased ipsilateral motor and somatosensory cortical activation during task performance would be positively associated with age and 2) increased activation would be either irrelevant to task performance or associated with poorer performance, supporting age-related dedifferentiation. Similarly, in terms of functional connectivity, we hypothesized that stronger positive inter and intrahemispheric connectivity between regions within the sensorimotor network would be 1) positively associated with age and 2) either irrelevant to task performance or negatively associated with motor and somatosensory task performance, also consistent with dedifferentiation. Given that previous animal and human studies have demonstrated that motor and somatosensory areas are densely interconnected, we also tested whether age differences in activity and connectivity between the two systems (i.e., motor and somatosensory) are related.

\section{Materials and Methods}

\section{Participants}

Thirty-seven healthy participants (21 females; 16 males) ranging in age from 19 to 76 years (mean $=43.7 \pm 17.3$ ) were recruited for this study. These participants were also included as part of a larger sample from our previous study in which we analyzed motor and somatosensory behavioral data (Ruitenberg et al. 2019). All participants were right-handed and had normal or corrected hearing and vision. Participants were screened to ensure that they were not taking any medications with psychotropic effects and were free from any MRI safety contraindications. They reported no history of psychiatric or neurological disorders. Motor behavioral data from four participants (ages 19, 20, 24, and 26) were excluded due to technical issues with the presentation/recording software during fMRI scanning. As such, the brain-behavior results for the motor task were based on the remaining 33 participants (18 females, 15 males; age range $22-76$; mean $=46.3 \pm 16.6$ ). All study procedures were reviewed and approved by the University of Michigan Institutional Review Board. Participants provided written informed consent before their involvement in the study.

\section{Experimental Procedures}

We performed testing over 1-2 sessions, depending on each participant's and the MRI scanner's availability. For participants that completed the testing over two sessions, the test days were separated by not more than one week. We administered the Montreal Cognitive Assessment (MoCA) (Nasreddine et al. 2005), the Digit Symbol Substitution Test (DSST) (McLeod et al. 1982), and the Purdue pegboard test (Desrosiers et al. 1995) (right hand, left hand, bimanual, and assembly tasks; three repetitions per task), to assess general cognitive and motor functioning. Participants also performed a series of tactile tasks outside of the scanner that assessed their tactile processing capacity. In addition, participants performed motor and (passive) somatosensory tasks while lying supine in the MRI scanner. We acquired structural MRI, resting state fMRI, and task-based fMRI data. Structural and resting state data are not presented in the current report. Taskbased fMRI data acquisition and analyses are described in detail below. 


\section{Tactile Behavioral Tasks}

Participants performed a battery of computerized tasks outside of the scanner that evaluated their general tactile processing capacity as well as their tactile intra and interhemispheric inhibitory function. This battery included 1) simple reaction time (RT); 2) choice RT; 3) static detection threshold (DT); 4) dynamic DT without simultaneous stimulation (baseline condition); 5) dynamic DT with simultaneous stimulation to another finger on the same hand (intrahemispheric condition); and 6) dynamic DT with simultaneous stimulation to a finger on the other hand (interhemispheric condition). Tactile stimulation was delivered via a CM4 four-digit vibrotactile stimulator (Cortical Metrics), developed at the University of North Carolina at Chapel Hill (Tommerdahl et al. 2010; Holden et al. 2012; Nguyen et al. 2014). All stimuli were delivered to the glabrous skin of the index and middle fingers using a cylindrical probe (5 $\mathrm{mm}$ in diameter).

The RT and DT tasks have been described in detail in previous studies (Zhang et al. 2011). In the RT tasks, participants received a vibration to one finger (duration of $40 \mathrm{~ms}$, amplitude of $200 \mu \mathrm{m}$, frequency of $25 \mathrm{~Hz}$ ) and were instructed to click a mouse button as soon as they felt it. In the simple RT task, any mouse click was sufficient (10 trials per finger on each hand), whereas in the choice RT task, participants were instructed to additionally indicate on which finger they felt the vibration by clicking either the left or right mouse button (five trials per finger on each hand). RT was recorded for each trial. In the choice RT task, response accuracy was also recorded. For both RT tasks, we determined the mean RTs across all trials for each participant. RTs that deviated more than three standard deviations from the mean of a participant were removed from the analyses. For the choice RT task, we also excluded trials with incorrect responses.

In the static DT task, participants received a vibration to either the index or middle finger of one hand (10 trials per finger) and were subsequently asked to indicate the finger on which the vibration was felt. For this task, the stimulus started at $15 \mu \mathrm{m}$ on the first trial and was adjusted on subsequent trials using an adaptive staircase algorithm that depended on the accuracy of the participant's response. The static threshold was defined as the smallest constant-amplitude stimulus that a participant could detect correctly.

In the dynamic DT task without simultaneous stimulation, participants were instructed to select the finger on which they felt a vibration as quickly as possible. The stimulus amplitude started at $0 \mu \mathrm{m}$ and then increased at a rate of $2 \mu \mathrm{m} / \mathrm{s}$. The stimulus amplitude at the time of each participant's response was recorded for each trial (seven trials per hand). Baseline DT was calculated for each participant as the minimum detectable amplitude of a stimulus that gradually increased in intensity starting from zero (Zhang et al. 2011).

For the inhibition tasks, participants were instructed to click a mini mouse button (placed under the subject's thumb; "TinyMouseT Optical"; Chester Creek Technologies, Inc.) as soon as they felt a vibration on their right index finger (R2). Similar to the baseline DT tasks, the target stimulus was delivered to R2 at a starting amplitude of $0 \mu \mathrm{m}$ and then increased at a rate of $2 \mu \mathrm{m} / \mathrm{s}$. At the same time, a conditioning stimulus was delivered to either the right middle finger (R3; intrahemispheric condition) or the left index finger (L2; interhemispheric condition). This conditioning stimulus was delivered at a frequency of $25 \mathrm{~Hz}$, with an amplitude of either 15, 50, 100, or $200 \mu \mathrm{m}$ (four trials per amplitude, randomly ordered). The intertrial interval was
$5 \mathrm{~s}$ and each trial began with a short delay period that did not include stimulation. To obtain individual DTs, we averaged the stimulus amplitude at the time of each participant's response on correct trials; this was performed as a function of conditioning amplitude $(15,50,100$, or $200 \mu \mathrm{m})$ and condition (intra or interhemispheric). To examine the effect of delivering a conditioning stimulus to either R3 or L2 on the detection of the target stimulus (R2), we compared R2 baseline performance (average DT on R2 trials without conditioning stimulus) with performance during $15,50,100$, or $200 \mu \mathrm{m}$ conditioning stimuli. We normalized the observed DTs to correct for individual differences in baseline performance by calculating the percent change compared to baseline for each of the conditioning amplitudes. To evaluate the effect of age on tactile performance, we performed regression analyses using age as the independent variable and DT (at baseline) or percent change as the dependent variables.

\section{fMRI Motor Tapping Task}

Participants viewed four horizontally aligned blank squares, two to the left and two to the right of a crosshair positioned in the center of the visual display, corresponding to the index and middle fingers of their hands. They were instructed to tap the corresponding finger as fast as possible once the square lit up with a (blue or orange) color, and to stay "in sync" with the colors lighting up for the duration of the block. After practicing one run of the task in the MRI control room, participants performed five different finger-tapping conditions in the scanner while fMRI and behavioral data were collected. In three conditions, participants were instructed to tap with a single finger (either R2, R3, or L2). In another condition, they were instructed to tap their right index finger followed by their right middle finger (R2R3; unimanual). In a final condition, they were instructed to tap their right index finger followed by their left index finger (R2L2; bimanual). The between finger lag was cued to be $200 \mathrm{~ms}$ by the flashing stimuli; thus the fingers tapped in a gallop fashion. The color of the squares represented whether the condition was a single- or two-finger condition (counterbalanced across participants).

Participants performed two runs of the motor task in which each of the five conditions occurred twice (randomly ordered). The 20-s experimental blocks were interleaved with 12-s rest blocks during which participants were instructed to gaze at the centrally presented crosshair. The intertrial interval was always $1 \mathrm{~s}$ and the interstimulus interval in the two-finger condition was $200 \mathrm{~ms}$. The stimulus presentation, timing, and response logging were controlled by Cogent 2000 software (http://www. vislab.ucl.ac.uk/cogent.php). We used two Fiber Optic Response Claws (one for each hand; Psychology Software Tools) to record participants' finger tapping responses.

Performance was assessed by calculating the mean betweenfinger "lag" and the variability of this lag across both runs in each condition. The between-finger lag was defined as the time between two consecutive taps with a single finger (averaged across the three conditions), or between the asynchronous taps with R2R3 or R2L2 (i.e., the intra and interhemispheric conditions, respectively). Variability of the between-finger lag was defined as the standard deviation (SD) of the lag. The SD reflects the ability of participants to maintain the $1 \mathrm{~s}$ lag in the single-finger conditions and the $200 \mathrm{~ms}$ lag in the intra and interhemispheric conditions. We excluded trials during which an error was made (i.e., participants tapped the wrong finger) or when the average between-finger lag or variability was 
more than 2.5 standard deviations from the group average (Fling et al. 2010). To evaluate the effect of age on motor tapping performance, we performed regression analyses using age as the independent variable and the between-finger lag or SD for each tapping measure as the dependent variable. These betweenfinger lag and SD measures were the final outcome measures used for participants in subsequent brain-behavior association analyses.

\section{fMRI Somatosensory Task}

Participants also performed two runs of a passive somatosensory task while lying supine in the MRI scanner. For this task, participants received vibrotactile stimulation to the index and/or middle fingers of one or both hands. The five conditions were identical to those used in the motor task (i.e., R2, R3, L2, R2R3, and R2L2 conditions), except stimulation was delivered simultaneously (not asynchronously) for the twofinger conditions. For this task, participants were instructed to gaze at a fixation cross in the center of the visual display while trying to focus on the sensation felt during each vibrotactile stimulation. Similar to the motor task, participants performed two runs of the somatosensory task, in which each of the five conditions occurred twice (randomly ordered) and lasted for 20-s experimental blocks interleaved with 12-s rest blocks. Tactile stimulation was delivered for $500 \mathrm{~ms}$ via an MRIcompatible CM4 four-digit vibrotactile stimulator (Cortical Metrics) (Tommerdahl et al. 2010; Holden et al. 2012; Nguyen et al. 2014) and followed by a $1500 \mathrm{~ms}$ delay period. Stimulus presentation and timing were controlled using Microsoft Visual Studio.

\section{MRI Data Acquisition}

Structural and functional brain images were acquired using a 3.0 Tesla GE MRI scanner, located at the University of Michigan Functional Magnetic Resonance Imaging Laboratory. For all participants, a 16-rod bird cage head coil was used and movement was minimized by using head cushions and Velcro straps. We obtained T1-weighted structural images, highresolution structural images using spoiled 3D gradient-echo acquisition (SPGR), and T2*-weighted functional images. Functional images were acquired using a single-shot gradient-echo (GRE) reverse spiral pulse sequence with the following parameters: field of view $=220 \times 200 \mathrm{~mm}$; voxel size $=3 \times 3 \times 4 \mathrm{~mm}(40$ axial slices); TR (repetition time) $=2 \mathrm{~s}$; TE (echo time) $=30 \mathrm{~ms}$.

\section{fMRI Data Preprocessing and Analysis}

Preprocessing of the task-based fMRI data was performed with the Statistical Parametric Mapping 8 software (SPM8; www.fil. ion.ucl.ac.uk/spm). Preprocessing steps included slice-time correction, realignment, segmentation of structural images, normalization into standard Montreal Neurological Institute (MNI) space, and spatial smoothing using a Gaussian kernel of $8 \mathrm{~mm}$ full width at half-maximum. We used the Artifact Detection Toolbox developed by the Gabrieli Lab at MIT (https://www.nitrc. org/projects/artifact_detect) to account for head motion artifacts in the MRI scanner. An image was considered an outlier if 1) head displacement in the $x, y$, or $z$ direction was greater than $0.5 \mathrm{~mm}$ from the previous frame; 2) the rotational displacement was greater than 0.02 radians from the previous frame; or 3 ) the global mean intensity of an image was greater than three standard deviations from the mean image intensity of the entire scan. We did not exclude any participants from analyses due to excess motion, as all participants had $<16 \%$ of outlier volumes, with an average of $1.3 \% \pm 0.45 \%$ motor outlier volumes and $0.9 \% \pm 0.43 \%$ tactile outlier volumes. Neither the percentage of motor nor tactile volumes correlated with age, $P s>0.27$. Outliers in the global mean signal intensity and motion were subsequently included as nuisance covariates in the first level general linear model (GLM).

A first-level GLM was conducted at an individual level across runs contrasting periods of finger tapping or vibrotactile stimulation with rest for the intra and interhemispheric conditions (i.e., R2R3 vs. rest and R2L2 vs. rest). The model included separate regressors for each of the experimental blocks convolved with a canonical hemodynamic response function. To examine the association between age and brain activation during these conditions, we performed second-level one-sample t-tests. In these models, first-level contrasts for each condition were included as the main variables of interest and age (in years) were included as a covariate. In order to explore regions exhibiting age-related differences in activity for more complex, multiple-finger conditions (e.g., R2R3) compared to simpler single-finger conditions (e.g., R2), we examined two additional statistical contrasts: R2L2 > R2 and R2R3 > R2. Statistical significance was determined by applying false discovery rate (FDR) $P<0.05$ at the cluster-level to correct for multiple comparisons.

To explore the relationship between performance and brain regions that showed an effect of age, Pearson's correlation coefficient was calculated between the average beta within the significant clusters and behavioral indices (e.g., betweenfinger lag). We also performed partial correlations (controlling for age) between these betas and performance to test whether age accounted for both neural and behavioral variance. In case we observed no association between age and brain activation for a given fMRI task, we examined the association between wholebrain activation and behavior by performing a one-sample t-test with SPM. To do this, we used the first-level contrasts for each condition (e.g., R2R3 tapping condition activation) as the main variable of interest and the corresponding behavioral measure (e.g., the R2R3 between-finger lag) as the covariate. For fMRI data obtained during the tactile task, we correlated this with tactile behavioral measures outside of the scanner (i.e., simple and choice RT, static and dynamic thresholds, \% change in DT with conditioning).

It is important to note that for the behavioral measures of inhibition (i.e., intra and interhemispheric tapping and tactile stimulation), we only assessed the relationship between corresponding brain and behavioral conditions. Given our hypotheses, we only assessed the relationship between R2R3 tapping/stimulation activity with the intrahemispheric tactile/motor behavioral measures and we only assessed the relationship between R2L2 tapping/stimulation activity with the interhemispheric tactile/motor behavioral measures.

\section{fcMRI Data Processing and Analysis}

In addition to the preprocessing steps above, we also performed additional denoising on the task-based fMRI data in preparation for fcMRI analysis using the CONN toolbox (Whitfield-Gabrieli and Nieto-Castanon 2012). The task-based fMRI data were filtered using a temporal high-pass filter of $0.008 \mathrm{~Hz}$. This filter was used for the task-based data instead of a typical band-pass filter of $0.008-0.09 \mathrm{~Hz}$ (as is used with resting state data) to 
avoid unnecessary smoothing over task boundaries. For additional noise reduction, we used the anatomical componentbased noise correction method (aCompCor), which models the influence of noise as a voxel-specific linear combination of several empirically estimated noise sources (Behzadi et al. 2007). This was done by deriving principal components from noise regions of interest (ROI) and including them as nuisance covariates in the first-level GLM. These noise ROIs (including white matter and cerebrospinal fluid masks) in addition to residual head movement parameters (including three rotations, three translations, and six parameters that reflect their first-order temporal derivatives) were regressed out during the calculation of functional connectivity maps.

First-level seed-to-voxel fcMRI analyses were also performed with the CONN toolbox. For these analyses, we created five seed ROIs (using $4 \mathrm{~mm}$-diameter spheres) based on the peak coordinates from the fMRI activation analyses (see fMRI results below). For the motor task-based fcMRI analysis, this included one region in right precentral gyrus, one region in right postcentral gyrus, and two regions in right lateral occipital cortex (LOC; which has been found to be involved in sensorimotor tasks (Astafiev et al. 2004; Peelen and Downing 2005; Zimmermann et al. 2016). For the tactile task-based fcMRI analysis, this included one region that contained left LOC, left somatosensory cortex, and left supramarginal gyrus (SMG). The mean time series of each seed region was obtained by averaging the time series of all voxels within that region. Next, Pearson's correlation coefficients were computed between the mean time series of each seed region and that of each voxel in an a priori brain mask. This mask is a standard whole-brain mask (in MNI space) used for voxel-based analyses and limits the analyses only to voxels within that mask. Correlation coefficients were then converted into $z$-values using Fisher's $r$-to- $z$ transformation (Zar 1996).

We next performed second-level GLMs to examine the association between age and functional connectivity between the seed ROIs and the rest of the brain. In these models, the first-level connectivity maps for each of the five conditions were included as the main variables of interest and age (in years) were included as a covariate. These models were performed separately for the motor and tactile tasks. Statistical significance was determined by applying FDR $P<0.05$ at the cluster-level to correct for multiple comparisons. To explore the relationship between performance and functional connectivity between brain regions that showed an effect of age, Pearson's correlation coefficient was calculated between the average connectivity within the significant clusters and motor (between-finger lag and SD) and tactile (RT, static threshold, DT without conditioning, percent change from 0 to $15 \mu \mathrm{m}$, and the slope across DT changes in the $15,50,100$, and $200 \mu \mathrm{m}$ conditions) performance. We also performed partial correlations (controlling for age) between these betas and performance to test whether age accounted for both neural and behavioral variance. Similar to the fMRI analyses, for the behavioral measures of inhibition (i.e., intra and interhemispheric tapping and tactile stimulation), we only assessed the relationship between R2R3 tapping/stimulation connectivity with the intrahemispheric tactile/motor behavioral measures and we only assessed the relationship between R2L2 tapping/stimulation connectivity with the interhemispheric tactile/motor behavioral measures. All brain-behavioral statistical analyses used a two-tailed level of 0.05 for defining statistical significance. Reported P-values were not corrected for multiple comparisons.
Table 1 Overview of participants' performance on the general cognitive and motor neuropsychological tests

\begin{tabular}{lcccc}
\hline & Mean & SD & Range & Relation with age \\
\hline MoCA & 27.8 & 1.5 & $24-30$ & $\mathrm{r}(37)=-.23 ; \mathrm{P}=0.17$ \\
PP right hand & 14.8 & 2.4 & $10.7-19$ & $\mathrm{r}(37)=-.56 ; \mathrm{P}<0.001$ \\
PP left hand & 13.8 & 2 & $9.7-17$ & $\mathrm{r}(37)=-.49 ; \mathrm{P}<0.01$ \\
PP both hands & 11.6 & 1.9 & $8.3-15.3$ & $\mathrm{r}(37)=-.41 ; \mathrm{P}<0.05$ \\
PP assembly & 34.5 & 7.8 & $17.7-48$ & $\mathrm{r}(37)=-.61 ; \mathrm{P}<0.001$ \\
DSST version 1 & 59.3 & 13.3 & $26-79$ & $\mathrm{r}(36)=-.75, \mathrm{P}<0.001$ \\
DSST version 2 & 59.4 & 12 & $28-77$ & $\mathrm{r}(36)=-.63, \mathrm{P}<0.001$ \\
\hline
\end{tabular}

\section{Results}

\section{General Cognitive and Motor Function}

Performance on the general cognitive and motor tests is summarized in Table 1. Correlation analyses demonstrated that manual motor performance as assessed with the Purdue Pegboard test declined with increasing age. In addition, information processing speed as assessed with the DSST declined with increasing age. However, general cognitive abilities as evaluated with the MoCA did not significantly change with age.

\section{Tactile Performance}

The results from the (out-of-scanner) tactile tasks were consistent with those reported in Ruitenberg et al. 2019; please refer to this paper for more detailed results and corresponding figures. Regression analyses with age as the independent variable and general tactile processing measures as the dependent variables showed that older age was associated with declines in RTs (simple: $B=1.67, t=3.16, P=0.003$; choice: $B=6.94, t=3.54, P=0.001$ ) as well as static thresholds $(B=0.11, t=5.34, P<0.001)$. Marginally, significant age declines were observed in baseline dynamic DTs $(B=0.13, t=2.02, P=0.051)$. We also found that age significantly predicted the \%change in DT for R2 from baseline when a 15- $\mu \mathrm{m}$ conditioning stimulus was added in both the interhemispheric $(B=-.905, t=-2.97, P=0.005)$ and intrahemispheric $(B=-.934$, $t=-2.79, P=0.009)$ conditions. Specifically, results showed that older age is associated with greater improvements (decreases) in DT when a $15 \mu \mathrm{m}$ conditioning stimulus is added, indicative of age-related declines in both inter and intrahemispheric inhibition.

\section{Motor Tapping Performance}

The results from the motor tapping task have been reported previously in our previous study (Ruitenberg et al. 2019). In short, we observed that age significantly predicted the intrahemispheric between-finger lag, such that increasing age was associated with an increased duration of the between-finger lag $(B=2.22$, $t=4.14, P<0.001)$. We also observed a marginally significant relationship between age and the interhemispheric betweenfinger lag $(B=1.06, t=2.01, P=0.054)$. We found no significant associations between age and any of the single-finger measures $(P s>0.42)$ or the two-finger SD measures (Ps $>0.34$ ).

Consistent with our previous study, we did not observe a significant correlation between performance of asynchronous movements and tactile inhibition for either intrahemispheric $(P s>0.13)$ or interhemispheric conditions (Ps $>0.35)$. 
Table 2 Associations between fMRI (motor) task-based activation and age

\begin{tabular}{llccc}
\hline Anatomical location & Contrast & Direction & Coordinates of peak & T-value \\
\hline R PreCG & R2R3 $>$ Rest & + & $34,-18,70$ & 5.29 \\
R PostCG & R2R3 > R2 & + & $4,-44,74$ & 4.26 \\
R LOC & R2R3 $>$ R2 & + & $26,-82,24$ & 795 \\
R LOC & R2L2 $>$ R2 & + & $34,-72,30$ & 3.01 \\
\hline
\end{tabular}

\section{Associations between fMRI and Age}

For the fMRI visuomotor task, we observed a significant positive relationship between age and activation in right precentral gyrus during the R2R3 tapping condition, such that older age was associated with greater recruitment of ipsilateral precentral gyrus during this unimanual tapping condition (Fig. 1A; Table 2). Results of the contrast of R2R3 > R2 revealed significant positive relationships between age and activation in right postcentral gyrus (Fig. 1B; Table 2) and right LOC (Fig. 1C; Table 2). Finally, results of the R2L2 > R2 contrast showed a significant positive relationship between age and activation in right LOC (Fig. 1D; Table 2). We did not observe any significant associations between age and neural activity during R2L2 tapping. We also did not observe any significant associations between age and neural activity during the fMRI tactile task. Thus, we did not test for a relationship between age differences in motor and somatosensory functional activation.

\section{Associations between fMRI and Behavior}

In terms of associations between performance and brain activity in regions that showed an effect of age in the motor task, we observed a significant positive relationship between activity in right precentral gyrus and the intrahemispheric betweenfinger lag, $r(33)=0.53, P<0.01$ (Fig. $2 A$ ). This effect was no longer significant after controlling for age, $r(30)=0.20, P=0.28$. Similarly, we observed a significant positive relationship between activity in right postcentral gyrus and the intrahemispheric betweenfinger lag, $r(33)=0.60, P<0.001$ (Fig. 2B). Again, this effect was no longer significant after controlling for age, $r(30)=0.31, P=0.09$. Finally, we observed a significant positive relationship between activity in right LOC and the intrahemispheric between-finger lag, $r(33)=0.52, P<0.01$ (Fig. $2 C$ ), but this effect was not significant after controlling for age, $r(30)=0.18, P=0.32$. We did not observe an association between LOC activation and the interhemispheric between-finger lag, $r(33)=0.18, P=0.32$. To confirm our findings using an omnibus statistical model within SPM, we entered performance as a covariate in the second level model and used an inclusive mask of the regions exhibiting an effect of age. We still observed the same significant associations between activation and behavior, Ps $<0.05$.

Because we did not observe an association between age and brain activity during the passive somatosensory task, we examined the association between whole-brain activation and tactile behavior (outside of the scanner) by performing a one-sample t-test with the behavioral data as covariates in SPM. We observed a significant negative relationship between activity in a cluster containing left LOC, postcentral gyrus, and SMG during R2R3 stimulation and simple RT (Fig. 3; Table 3). We did not observe any association between whole-brain activation and static/dynamic DTs, or DTs with conditioning.
Table 3 Associations between fMRI (tactile) task-based activation and tactile performance

\begin{tabular}{llcccc}
\hline $\begin{array}{l}\text { Anatomical } \\
\text { location }\end{array}$ & Contrast & $\begin{array}{c}\text { Direction } \\
\text { Coordinates } \\
\text { of peak }\end{array}$ & $\begin{array}{c}\text { T-value } \\
\text { L LOC, }\end{array}$ & $\begin{array}{c}\text { R2R3 } \times \\
\text { size }\end{array}$ \\
\hline $\begin{array}{l}\text { PostCG, } \\
\text { SMG }\end{array}$ & SRT & - & $-58,-46,28$ & 4.32 & 517 \\
\hline
\end{tabular}

\section{Associations between fcMRI and Age}

For the motor task, we observed that older age was associated with stronger positive functional connectivity between right precentral gyrus seed region and right LOC during the R2R3 tapping condition (Fig. 4A; Table 4). We also observed that older age was associated with weaker functional connectivity between left LOC seed region and right superior frontal gyrus (SFG; Fig. 4B; Table 4) during the R2R3 tapping condition. For the tactile task, we found that older age was associated with stronger positive functional connectivity between left LOC/S1/SMG seed region and right LOC/superior parietal lobule (SPL) during R2R3 vibrotactile stimulation (Fig. 4C; Table 4).

Given that age was positively associated with connectivity between similar sensorimotor regions (i.e., LOC, S1) during both the motor and somatosensory tasks, we explored whether such age effects in somatosensory neural representations are related to those in the motor system by extracting the connectivity betas from the suprathreshold clusters and performing bivariate correlations on these measures. We observed a significant positive relationship between the tactile connectivity betas between right and left LOC (during vibrotactile stimulation) and the motor connectivity betas between right precentral gyrus and LOC (during motor tapping; $r(37)=0.47, P<0.005$; Fig. $5 \mathrm{~A})$. This effect was no longer significant after controlling for age, $r(34)=0.13, P=0.45$. We observed a significant negative relationship between the same tactile connectivity betas and the motor connectivity betas between right LOC and SFG (during motor tapping; $r(37)=.-49, \mathrm{P}<0.005$; Fig. 5B). Again, this effect was no longer significant after controlling for age, $r(34)=-.11, P=0.54$.

\section{Associations between fcMRI and Behavior}

In terms of associations between motor tapping performance and functional connectivity between brain regions that showed an effect of age, we observed that stronger positive functional connectivity between right precentral gyrus seed region and ipsilateral LOC (during R2R3 tapping) was associated with an increased duration of the intrahemispheric between-finger lag, $r(33)=0.40, P=0.02$ (Fig. 6A). This effect was no longer significant after controlling for age, $r(30)=0.05, P=0.79$. We also observed a negative relationship between functional 
A

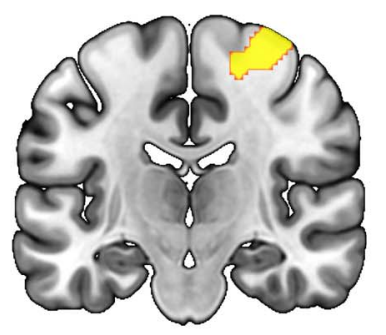

B

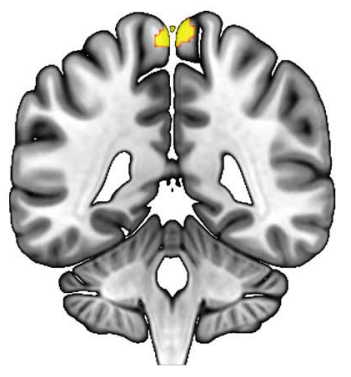

C

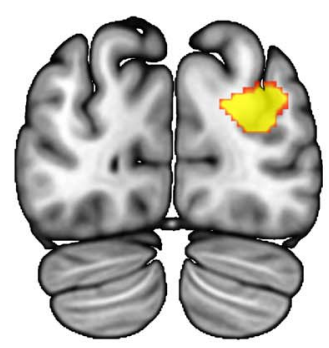

D

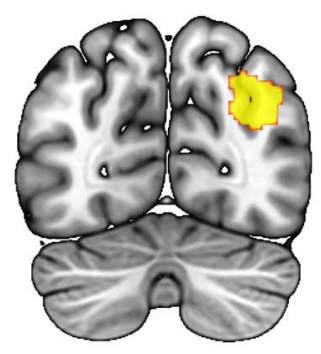

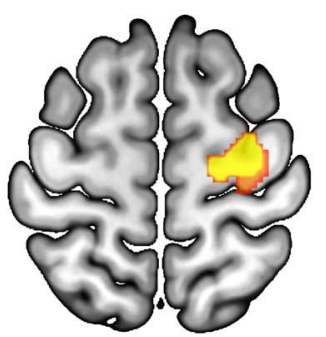
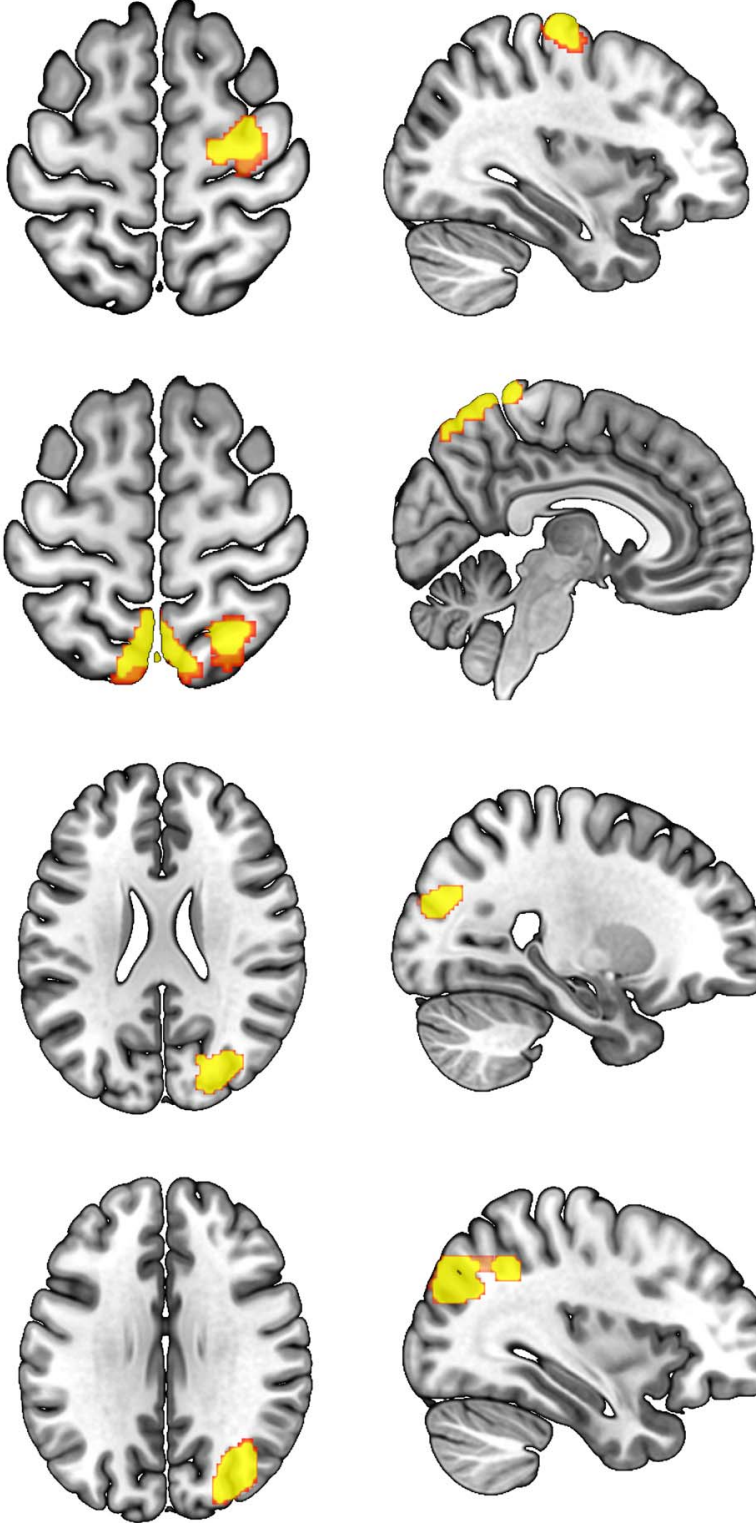

Figure 1. Associations between age and fMRI activation. (A) Older age was associated with greater recruitment of ipsilateral precentral gyrus during the unimanual (R2R3) tapping condition. Compared to a simpler single-finger condition (R2), a more complex two-finger condition (R2R3) elicited greater recruitment of ipsilateral (B) postcentral gyrus and (C) LOC with older age. (D) Compared to a simpler single-finger condition (R2), a more complex bimanual two-finger condition (R2L2) elicited greater recruitment of right LOC with older age. Numbers under brain images represent MNI coordinates of suprathreshold clusters.

Table 4 Associations between functional connectivity and age

\begin{tabular}{lllcccc}
\hline Seed region & Target region & Contrast & Direction & Coordinates of peak & T-value & Cluster size \\
\hline R PreCG & R LOC & Motor R2R3 > Rest & + & $36,-54,42$ & 5.4 & 372 \\
R LOC & R SFG & Motor R2R3 > Rest & - & $4,20,58$ & 6.72 & 237 \\
L LOC, PostCG, SMG & R LOC, SPL & Tactile R2R3 > Rest & + & $32,-54,44$ & 4.38 & 221 \\
\hline
\end{tabular}

connectivity between right LOC seed region and ipsilateral SFG and the intrahemispheric between-finger lag, $r(33)=-.43$, $P<0.01$ (Fig. 6B). Again, this effect was no longer significant after controlling for age, $r(30)=0.01, P=0.95$.

For tactile performance, we observed a positive relationship between functional connectivity between left LOC/S1/SMG seed region and right LOC/SPL (during R2R3 vibrotactile stimulation) and both simple and choice RTs (Fig. 7A,B, respectively), $r s(37)>0.43$, Ps $<0.008$. Controlling for age, the relationship between connectivity and simple RT was no longer significant, $r(34)=0.21, P=0.22$, whereas the relationship between connectivity and choice RT remained significant, $r(34)=0.49, P=0.003$. 
A

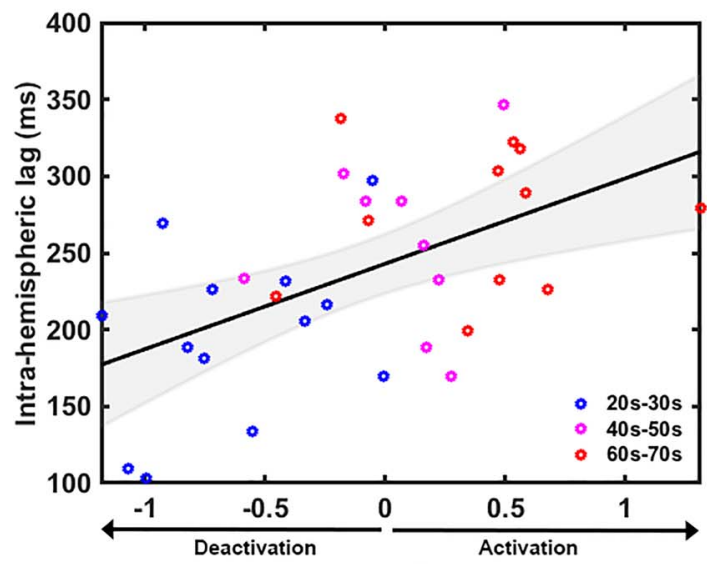

B

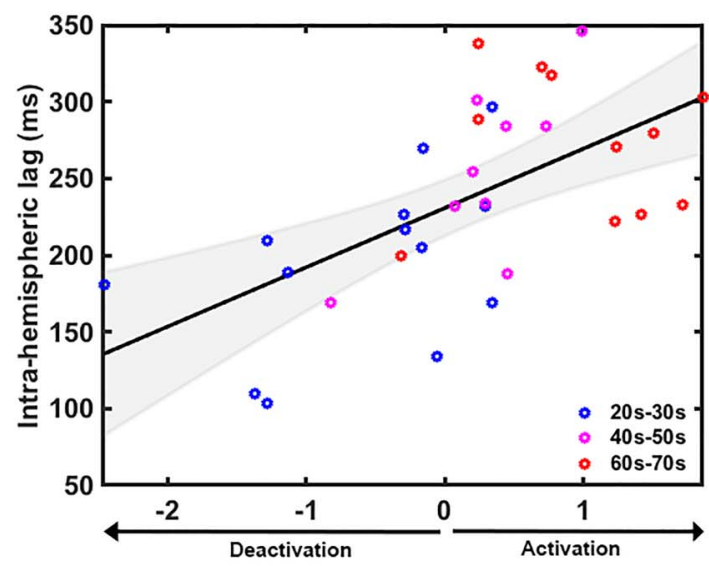

C

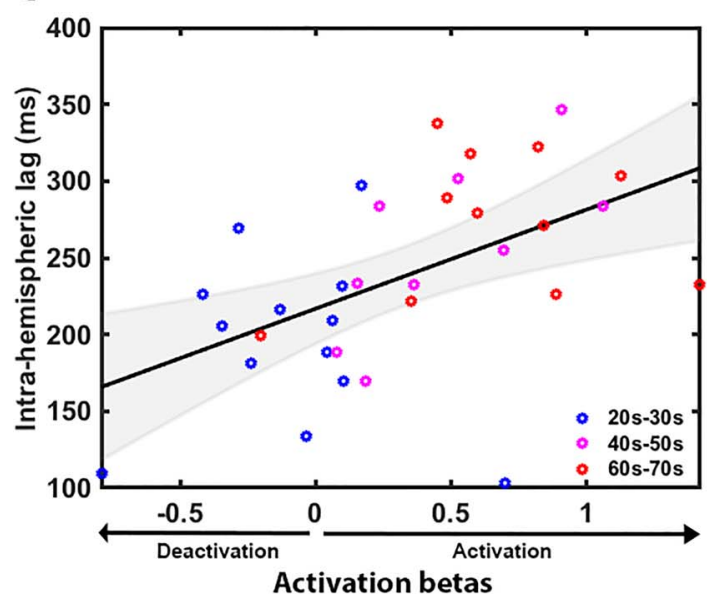

Figure 2. Associations between fMRI activation and motor performance. Increased ipsilateral activation in right (A) precentral gyrus (M1), (B) postcentral gyrus (S1), and (C) lateral occipital cortex (LOC) during R2R3 tapping was associated with an increased duration of the between-finger lag. Blue markers represent participants in their 20s-30s, magenta colors represent participants in their 40s-50s, and red colors represent participants in their 60s-70s. Shaded areas represent the $95 \% \mathrm{CI}$.

We did not observe any associations between connectivity during tactile stimulation and static/dynamic DTs, or DTs with conditioning, Ps $>0.06$

\section{Discussion}

In this study, we provide novel evidence regarding the relationship between healthy aging and neural dedifferentiation in both motor and somatosensory systems. Moreover, our findings suggest that dedifferentiation in these two systems is related. Below, we first discuss our findings for the motor and somatosensory systems separately and subsequently discuss the association between both.

\section{Evidence for Age-Related Neural Dedifferentiation in the Motor System}

Consistent with our hypothesis, the present results revealed that older age was associated with greater recruitment of ipsilateral motor cortex during a unimanual (i.e., R2R3) tapping condition. Furthermore, greater activation (i.e., positive activation compared to deactivation) was associated with worse motor performance (i.e., longer lags during the intrahemispheric tapping condition). These results are in line with previous findings demonstrating significant age differences between young and older adults in the distinctiveness of motor cortical representations. For instance, Carp et al. employed multivoxel pattern analysis to demonstrate that older adults had significantly less distinct motor cortical activation patterns compared to young adults (Carp et al. 2011). Similarly, Bernard and colleagues used TMS to demonstrate that older adults exhibit less distinct intracortical motor representations than young adults (Bernard and Seidler 2012). The present study extends these findings by providing novel evidence for neural dedifferentiation within the motor cortex across the lifespan.

We also found that compared to simple single-finger conditions, more complex two-finger conditions (both unimanual and bimanual tapping) elicited greater recruitment of right somatosensory and lateral occipital cortices with older age. Moreover, greater activation was associated with worse performance. One interpretation of these results is that with older age, declines in inhibition require participants to rely more on sensory regions and not just motor cortex (i.e., S1 and LOC) for more complex (asynchronous two-digit) tasks compared to simpler (one-digit) tasks. However, greater reliance on these sensory regions is associated with worse performance on the visuomotor task. Consistent with this notion, several studies have found that this region in LOC is involved in visuomotor actions (Astafiev et al. 2004; Peelen and Downing 2005; Zimmermann et al. 2016) and that attention modulates neural selectivity in this region (Murray and Wojciulik 2004). Thus, it could be that with older age, participants rely more on this dorsal visual pathway for more complex (e.g., requiring more attention to visual stimuli) motor tasks, but that individuals who do not rely as much on these regions perform better.

The functional connectivity results from the tapping task provide further evidence for age-related neural dedifferentiation in the motor system. Specifically, we found that older age was associated with stronger positive connectivity between ipsilateral motor cortex and LOC and that stronger positive connectivity (compared to negative connectivity) between these regions was associated with worse motor performance (i.e., longer lag times). It is possible that the activation and connectivity results stem from the same underlying mechanism because both findings indicate that regions in ipsilateral M1 and LOC (and functional connectivity between these areas) are impacted by age. Previous studies have also shown that both task-based 


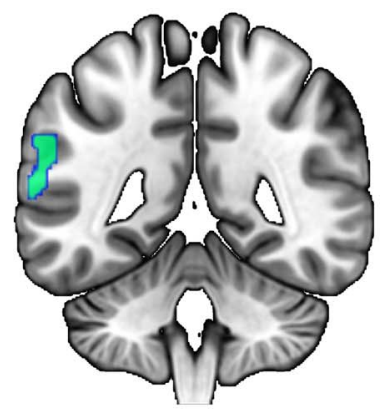

$-46$

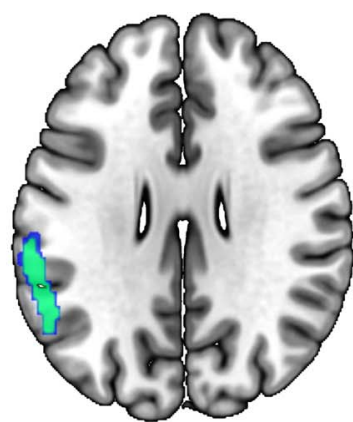

28

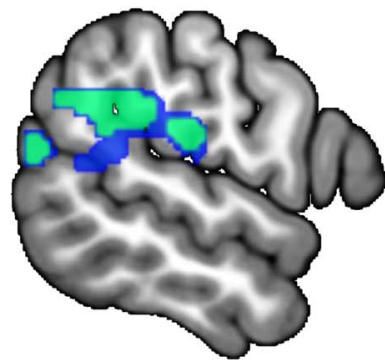

$-58$

Figure 3. Associations between fMRI activation and vibrotactile performance outside of the scanner. Increased activation in contralateral LOC, postcentral gyrus, and SMG during R2R3 vibrotactile stimulation was associated with faster tactile simple RTs.

A

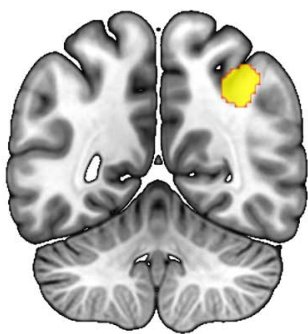

$-54$

B

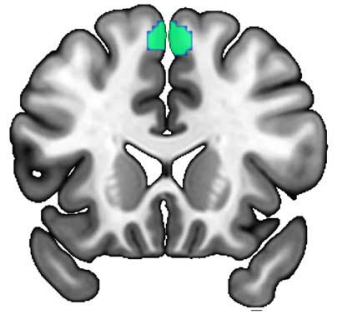

20

C

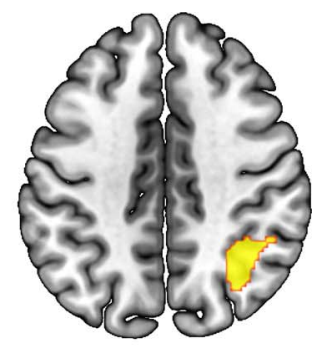

42

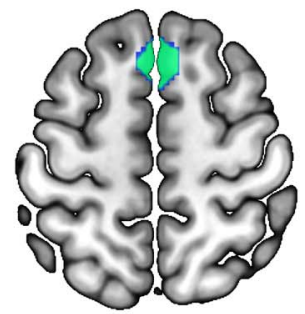

58

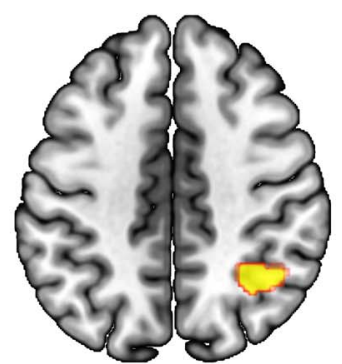

44

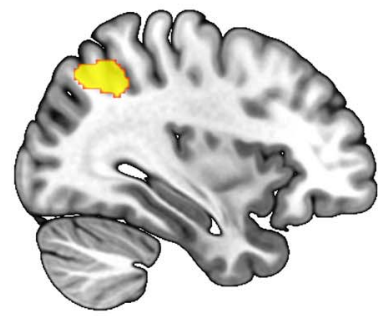

36

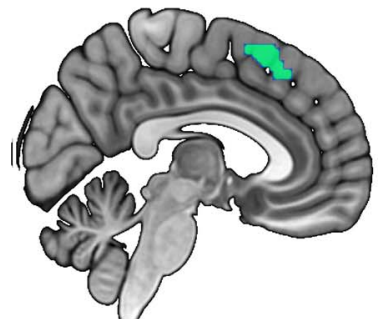

4

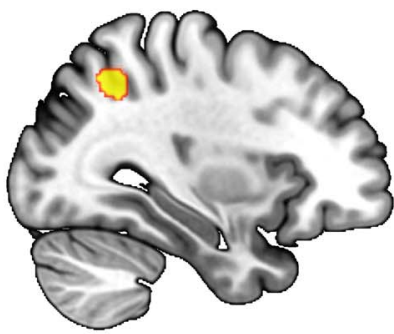

32

Figure 4. Associations between age and task-based functional connectivity. (A) Older age was associated with positive connectivity between ipsilateral precentral gyrus seed region and lateral occipital cortex (LOC) during R2R3 tapping. (B) Older age was associated with negative connectivity between ipsilateral LOC seed region and superior frontal gyrus during R2R3 tapping. Older age was associated with positive connectivity between left LOC seed region and (C) right LOC during R2R3 vibrotactile stimulation. Yellow colors represent positive associations; green colors represent negative associations.

and resting state functional connectivity and activation levels are correlated. For instance, Chan and colleagues found that individual differences in functional connectivity were related to differences in brain activity during two independent (visual and semantic) tasks (Chan et al. 2017). Moreover, Langan and colleagues showed that reduced interhemispheric resting state connectivity in older adults was related to a decreased ability to inhibit activity in the nondominant hemisphere during unimanual motor performance (Langan et al. 2010).

One present finding that is not consistent with dedifferentiation in the motor system is that older age was associated with stronger negative connectivity between right LOC and superior frontal gyrus during R2R3 tapping and that stronger positive connectivity between these regions predicted 
A

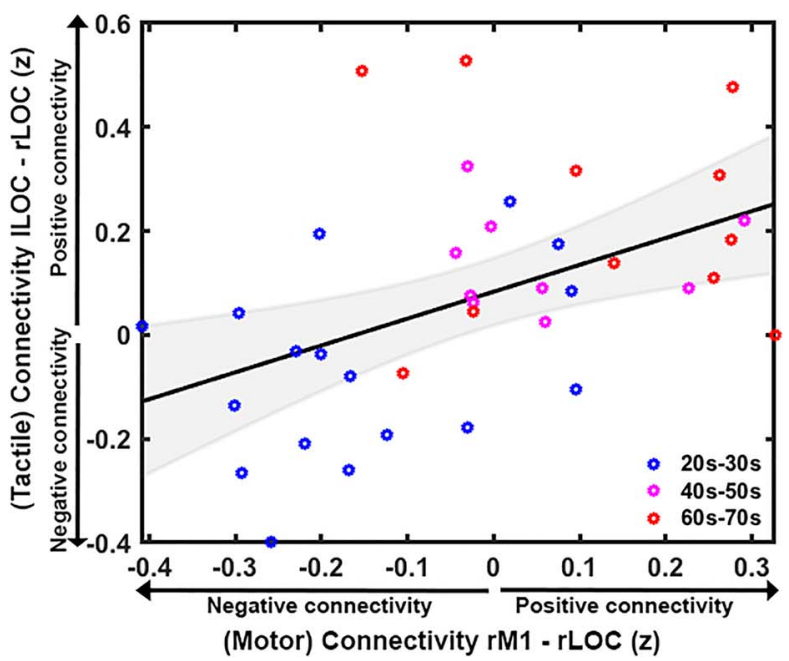

B

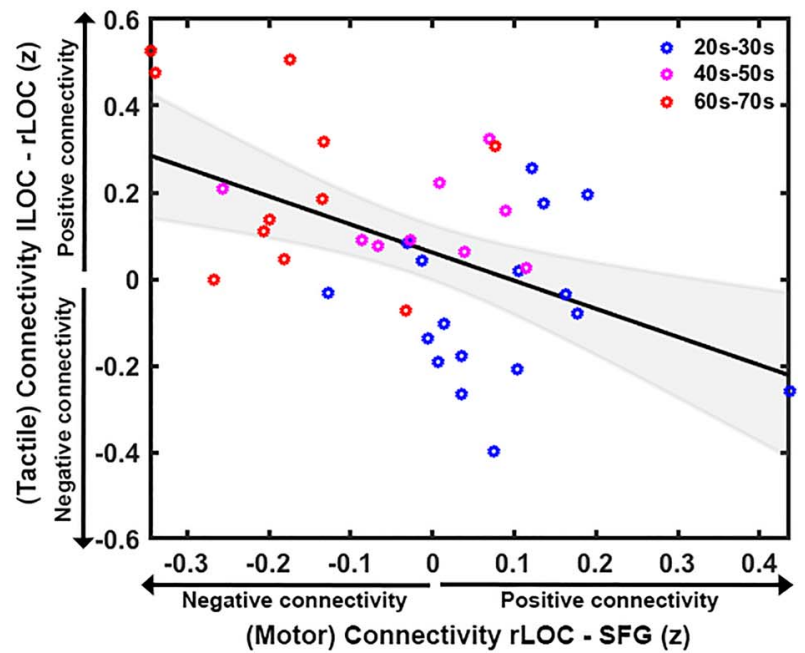

Figure 5. Associations between connectivity during tactile and motor fMRI tasks. (A) Stronger positive connectivity between right precentral gyrus and right LOC during R2R3 motor tapping was associated with stronger positive connectivity between left LOC and right LOC during R2R3 tactile stimulation. (B) Stronger positive connectivity between right LOC and superior frontal gyrus during R2R3 motor tapping was associated with stronger negative connectivity between left LOC and right LOC during R2R3 tactile stimulation.

A

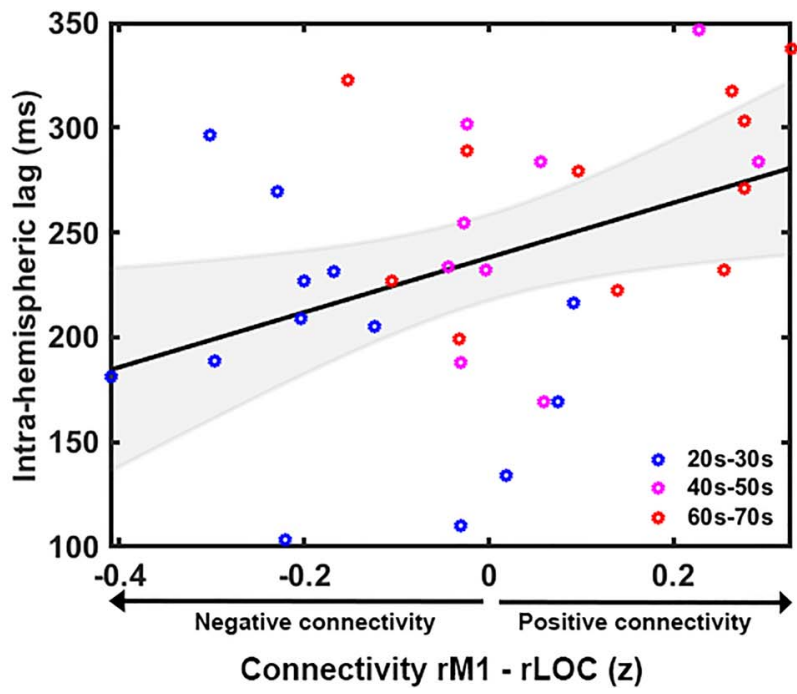

B

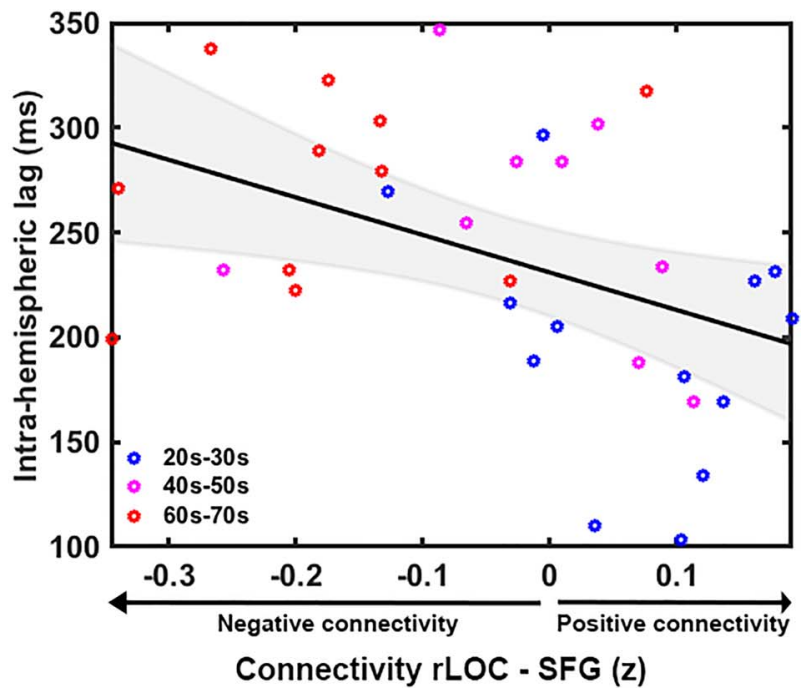

Figure 6. Associations between (motor) task-based functional connectivity and motor performance. (A) Stronger positive connectivity between ipsilateral precentral gyrus seed region and lateral occipital cortex (LOC) during R2R3 tapping condition was associated with an increased duration of the between-finger lag. (B) Stronger positive connectivity between ipsilateral LOC seed region and superior frontal gyrus during the intrahemispheric tapping condition was associated with a decreased duration of the between-finger lag.

better performance (i.e., shorter lag times). This finding is consistent with previous studies that have found that the recruitment of additional frontal regions serves a compensatory role for older adults performing motor actions. For instance, Heuninckx et al. 2008 reported a positive association between activation in higher-level sensorimotor and frontal regions and motor performance in older adults. Thus, it may be that the recruitment of higher level association regions (such as superior frontal cortex) provides compensation for sensorimotor neural dedifferentiation and other deleterious age effects on brain function.

\section{Evidence for Age-Related Neural Dedifferentiation in the Somatosensory System}

In the tactile task, we found that increased contralateral sensorimotor cortical (including LOC, S1, and SMG) activation during vibrotactile stimulation was associated with better performance (i.e., reduced simple RTs) across all participants. This finding is consistent with previous studies demonstrating robust contralateral somatosensory cortical activity during vibrotactile stimulation in healthy young adults (Hlushchuk and Hari 2006). The present study extends these findings 
A

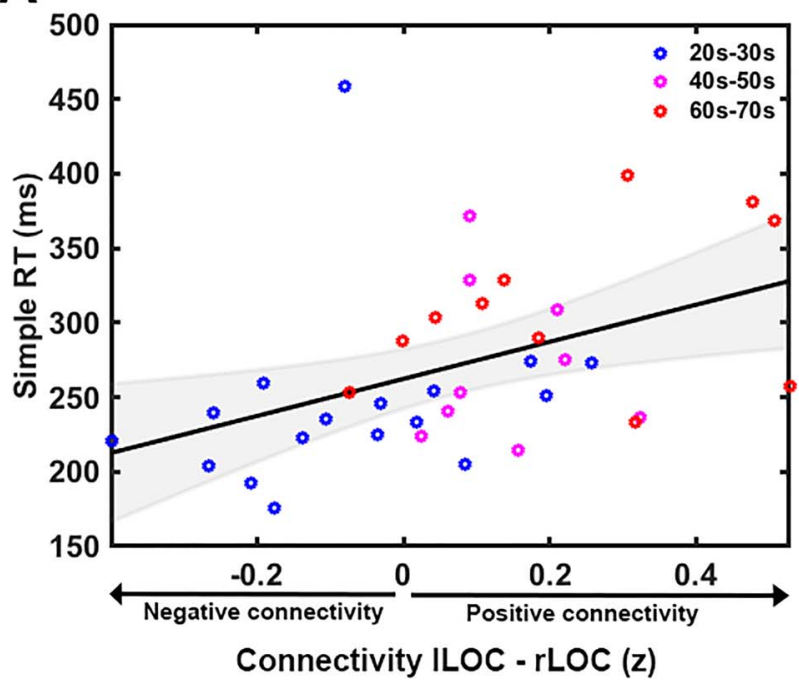

B

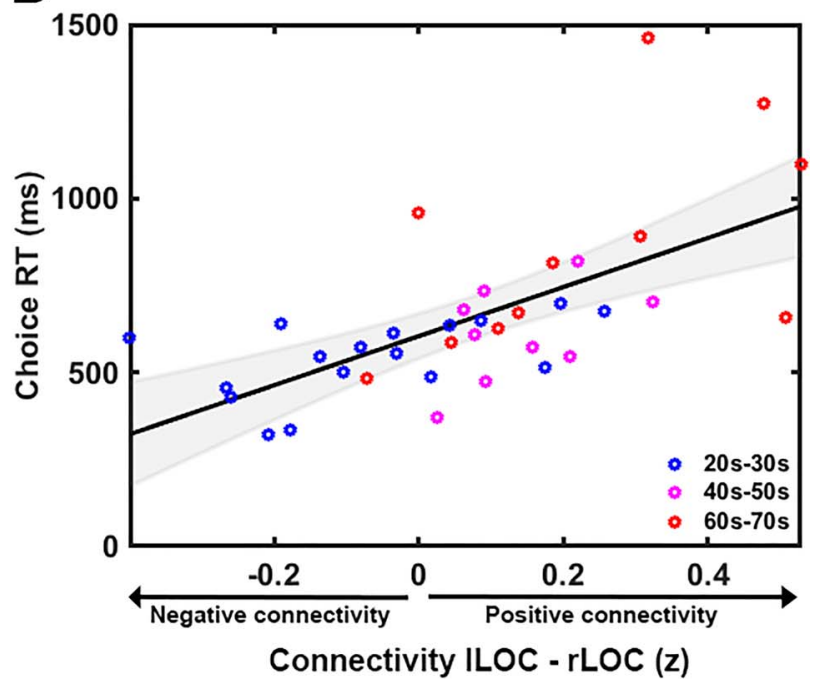

Figure 7. Associations between (tactile) task-based functional connectivity and vibrotactile performance. Stronger positive connectivity between left LOC seed region with right LOC during R2R3 stimulation was associated with slower (A) simple and (B) choice RTs.

by suggesting that greater contralateral activity (and thus inhibition of ipsilateral activity) in sensorimotor cortex is associated with better performance, independent of age.

We also found that older age was associated with stronger positive connectivity between these sensorimotor regions with right LOC and SPL during vibrotactile stimulation and that stronger interhemispheric positive connectivity between these areas predicted worse performance, indicative of dedifferentiation. These findings are consistent with previous work indicating that increased activity in ipsilateral sensorimotor cortex is associated with worse tactile performance (Lenz et al. 2012; Brodoehl et al. 2013; Pleger et al. 2016). For instance, Pleger and colleagues found that aging leads to tactile degradation that is related to enhanced fMRI activity in somatosensory cortex. The present study extends these previous findings by demonstrating that age-related increases in sensorimotor interhemispheric functional connectivity are detrimental to somatosensory performance. Furthermore, our results provide novel evidence that somatosensory neural representations differ across the adult lifespan.

\section{Age Differences in Somatosensory System Are Related to Age Differences in Motor System}

Our findings revealed that age differences in motor representations are related to age differences in somatosensory representations. Specifically, we observed a positive relationship between the tactile connectivity betas between right and left LOC (during vibrotactile stimulation) and the motor connectivity betas between right precentral gyrus and LOC (during visuomotor tapping). Although previous studies have investigated either motor or somatosensory dedifferentiation separately, this is the first study to explore both as well as the relationship between the two. Our results are in line with previous investigations in healthy young adults demonstrating overlapping cortical activity during motor and vibrotactile tasks (Gelnar et al. 1999; Arce-McShane et al. 2016). For instance, Gelnar and colleagues reported overlapping activity in precentral, postcentral, and premotor cortices as well as in SMA during both motor tapping and vibrotactile stimulation. Our study extends these findings by demonstrating that similar regions (i.e., LOC, S1) and the functional connectivity between them are impacted by age during both motor and somatosensory performance and that these age effects in the two systems are related.

It is perhaps not surprising that we observed a relationship between motor and somatosensory connectivity, given that numerous animal and human studies have demonstrated that motor and somatosensory areas are densely interconnected (White and DeAmicis 1977; Donoghue and Parham 1983; Veinante and Deschênes 2003). These cortical connections are thought to modulate the relationship between motor and sensory components of sensorimotor processes (Petreanu et al. 2009; Xu et al. 2012). The novel aspect of our finding is that the relationship between these systems was largely driven by age, indicating that dedifferentiation in the motor system is related to dedifferentiation in the somatosensory system.

Although we observed a relationship between age differences in motor and somatosensory neural representations in the current study, we did not observe a relationship between motor and tactile behavioral measures, either in the present study or in our previous study (Ruitenberg et al. 2019). One reason for this discrepancy between the neural and behavioral relationships may be that tactile stimulation during fMRI scanning was passive, whereas the (outside of scanner) tactile behavioral tasks were active. Thus, activation induced from passive tactile stimulation (in the scanner) could represent a different phenomenon from the active tactile behavioral measures collected outside of the scanner. Relatedly, the tactile stimulation in the behavioral task was different for the target and conditioning fingers. In contrast, for the fMRI task, both fingers received the exact same stimulation at the same time. This could also affect activation patterns and explain the differences in findings between the two studies.

\section{Limitations}

Several limitations constrain the interpretation of the present results. First, our cross-sectional sample only allows us to draw 
inferences about age differences and not longitudinal changes with age. Similarly, our correlation analyses can only be used to infer associations between measures, but cannot determine directionality. Future longitudinal designs would make it possible to determine the order of certain age-related changes, which may help to establish causal relationships. Furthermore, because all three variables of interest (i.e., age, brain, and behavioral measures) in this study are strongly associated, it is difficult to determine the precise relationships between any two given variables independent of the influence of the third variable (Damoiseaux 2017; King et al. 2018). For instance, after controlling for age while examining the relationships between brain and behavioral measures in this study, we observed (almost) no significant brain-behavior relationships. Given the strong associations between age and behavior in addition to age and connectivity (correlations above 0.6), it would be difficult to demonstrate a statistically significantly relationship between connectivity and behavior above and beyond the influence of age. Longitudinal studies are necessary to mitigate these issues and permit the assessment of age-related changes and the relationship between brain and behavioral measures independent of age.

Another limitation in the current study is that the older adults (and younger adults) who participated in this study represent a relatively high functioning population. Therefore, caution should be taken in generalizing the findings to a broader population. Future studies should aim to also evaluate changes in neural representations associated with pathological aging.

\section{Conclusions}

The present study provides novel evidence for neural dedifferentiation across the adult lifespan in both motor and somatosensory systems and suggests that dedifferentiation in these two systems is related. The findings from this study advance our understanding of the neural mechanisms that underlie agerelated declines in sensorimotor functioning. These findings may help lead to the development of targeted interventions and treatments to combat age-related sensorimotor behavioral decline.

\section{Funding}

National Institutes of Health (grant number NIH 1R56AG043402 awarded to R.D.S.); predoctoral fellowship from the National Institute on Aging and the National Institutes of Health (F31AG057205 to K.C.); postdoctoral fellowship from the Ghent University Special Research Fund (BOF 15/PDO/135 to M.F.L.R.).

\section{Notes}

We would like to thank Kyle Latack for assistance with data collection. We would also like to thank Jameson Holden for providing technical assistance with the vibrotactile stimulation device. M.T. is the cofounder of Cortical Metrics, a company licensed by the University of North Carolina to distribute devices that supported the methods reported in this paper.

Conflict of Interest. None declared.

\section{References}

Arce-McShane FI, Ross CF, Takahashi K, Sessle BJ, Hatsopoulos NG. 2016. Primary motor and sensory cortical areas communicate via spatiotemporally coordinated networks at multiple frequencies. Proc Natl Acad Sci. 113:5083-5088.

Astafiev SV, Stanley CM, Shulman GL, Corbetta M. 2004. Extrastriate body area in human occipital cortex responds to the performance of motor actions. Nat Neurosci. 7:542-548.

Behzadi Y, Restom K, Liau J, Liu TT. 2007. A component based noise correction method (CompCor) for BOLD and perfusion based fMRI. NeuroImage. 37:90-101.

Bernard JA, Seidler RD. 2012. Evidence for motor cortex dedifferentiation in older adults. Neurobiol Aging. 33:1890-1899.

Brodoehl S, Klingner C, Stieglitz K, Witte OW. 2013. Agerelated changes in the somatosensory processing of tactile stimulation-an fMRI study. Behav Brain Res. 238:259-264.

Cabeza R. 2002. Hemispheric asymmetry reduction in older adults: the HAROLD model. Psychol Aging. 17:85-100.

Cabeza R, Albert M, Belleville S, Craik FIM, Duarte A, Grady CL, Lindenberger U, Nyberg L, Park DC, Reuter-Lorenz PA et al. 2018. Maintenance, reserve and compensation: the cognitive neuroscience of healthy ageing. Nat Reu Neurosci. 19:701.

Carp J, Park J, Hebrank A, Park DC, Polk TA (2011) Age-related neural dedifferentiation in the motor system. PLoS One. 6. Available at: //WOS:000299684700053.

Chan MY, Alhazmi FH, Park DC, Savalia NK, Wig GS. 2017. Resting-state network topology differentiates task signals across the adult life span. J Neurosci. 37:2734-2745.

Damoiseaux JS. 2017. Effects of aging on functional and structural brain connectivity. Neuroimage. 160:32-40.

Desrosiers J, Hebert R, Bravo G, Dutil E. 1995. The Purdue pegboard test - normative data for people aged 60 and over. Disabil Rehabil. 17:217-224.

Donoghue JP, Parham C. 1983. Afferent connections of the lateral agranular field of the rat motor cortex. J Comp Neurol. 217:390-404.

Fling BW, Seidler RD. 2012. Task-dependent effects of interhemispheric inhibition on motor control. Behav Brain Res. 226:211-217.

Fling BW, Walsh CM, Bangert AS, Reuter-Lorenz PA, Welsh RC, Seidler RD. 2010. Differential callosal contributions to bimanual control in young and older adults. J Cogn Neurosci. 23:2171-2185.

Fujiyama H, Hinder MR, Schmidt MW, Garry MI, Summers JJ. 2012. Age-related differences in corticospinal excitability and inhibition during coordination of upper and lower limbs. Neurobiol Aging. 33:1484.e1-1484.e14.

Gelnar PA, Krauss BR, Sheehe PR, Szeverenyi NM, Apkarian AV. 1999. A comparative fMRI study of cortical representations for thermal painful, vibrotactile and motor performance tasks. NeuroImage. 10:460-482.

Heuninckx S, Wenderoth N, Debaere F, Peeters R, Swinnen SP. 2005. Neural basis of aging: the penetration of cognition into action control. J Neurosci. 25:6787-6796.

Heuninckx S, Wenderoth N, Swinnen SP. 2008. Systems neuroplasticity in the aging brain: recruiting additional neural resources for successful motor performance in elderly persons. J Neurosci. 28:91-99.

Hlushchuk Y, Hari R. 2006. Transient suppression of ipsilateral primary somatosensory cortex during tactile finger stimulation. J Neurosci Off J Soc Neurosci. 26:5819-5824. 
Holden JK, Nguyen RH, Francisco EM, Zhang Z, Dennis RG, Tommerdahl M. 2012. A novel device for the study of somatosensory information processing. J Neurosci Methods. 204: 215-220.

King BR, van Ruitenbeek P, Leunissen I, Cuypers K, Heise K-F, Santos Monteiro T, Hermans L, Levin O, Albouy G, Mantini $D$ et al. 2018. Age-related declines in motor performance are associated with decreased segregation of large-scale resting state brain networks. Cereb Cortex. 1-13.

Langan J, Peltier SJ, Bo J, Fling BW, Welsh RC, Seidler RD. 2010. Functional implications of age differences in motor system connectivity. Front Syst Neurosci. 4:17.

Lenz M, Tegenthoff M, Kohlhaas K, Stude P, Hoffken O, Tossi MAG, Kalisch T, Dinse HR. 2012. Increased excitability of somatosensory cortex in aged humans is associated with impaired tactile acuity (vol 32, pg 1811, 2012). J Neurosci. 32:6411-6411.

Mattay VS, Fera F, Tessitore A, Hariri AR, Das S, Callicott JH, Weinberger DR. 2002. Neurophysiological correlates of age-related changes in human motor function. Neurology. 58:630-635.

McLeod DR, Griffiths RR, Bigelow GE, Yingling J. 1982. An automated version of the digit symbol substitution test (DSST). Behav Res Methods Instrum. 14:463-466.

Murray SO, Wojciulik E. 2004. Attention increases neural selectivity in the human lateral occipital complex. Nat Neurosci. 7:70-74.

Nasreddine ZS, Phillips NA, Bedirian V, Charbonneau S, Whitehead V, Collin I, Cummings JL, Chertkow H. 2005. The Montreal cognitive assessment, MoCA: a brief screening tool for mild cognitive impairment. J Am Geriatr Soc. 53:695-699.

Nguyen RH, Forshey TM, Holden JK, Francisco EM, Kirsch B, Favorov O, Tommerdahl M. 2014. Vibrotactile discriminative capacity is impacted in a digit-specific manner with concurrent unattended hand stimulation. Exp Brain Res. 232:3601-3612.

Park J, Carp J, Hebrank A, Park DC, Polk TA. 2010. Neural specificity predicts fluid processing ability in older adults. J Neurosci. 30:9253-9259.

Peelen MV, Downing PE. 2005. Is the extrastriate body area involved in motor actions? Nat Neurosci. 8:125.

Peinemann A, Lehner C, Conrad B, Siebner HR. 2001. Agerelated decrease in paired-pulse intracortical inhibition in the human primary motor cortex. Neurosci Lett. 313: 33-36.

Petreanu L, Mao T, Sternson SM, Svoboda K. 2009. The subcellular organization of neocortical excitatory connections. Nature. 457:1142-1145.
Pleger B, Wilimzig C, Nicolas V, Kalisch T, Ragert P, Tegenthoff M, Dinse HR. 2016. A complementary role of intracortical inhibition in age-related tactile degradation and its remodelling in humans. Sci Rep. 6:27388.

Reuter-Lorenz PA. 2002. New visions of the aging mind and brain. Trends Cogn Sci. 6:394-400.

Reuter-Lorenz PA, Lustig C. 2005. Brain aging: reorganizing discoveries about the aging mind. Curr Opin Neurobiol. 15:245-251.

Riecker A, Gröschel K, Ackermann H, Steinbrink C, Witte O, Kastrup A. 2006. Functional significance of age-related differences in motor activation patterns. NeuroImage. 32:1345-1354.

Ruitenberg MF, Cassady KE, Reuter-Lorenz P, Tommerdahl M, Seidler RD (2019) Age-related reductions in tactile and motor inhibitory function start early but are independent. Front Aging Neurosci 11. https:/www.frontiersin.org/ articles/10.3389/fnagi.2019.00193/abstract (last accessed 24 July 2019).

Seidler RD, Bernard JA, Burutolu TB, Fling BW, Gordon MT, Gwin JT, Kwak Y, Lipps DB. 2010. Motor control and aging: links to age-related brain structural, functional and biochemical effects. Neurosci Biobehav Rev. 34:721-733.

Tommerdahl M, Favorov OV, Whitsel BL. 2010. Dynamic representations of the somatosensory cortex. Neurosci Biobehav Rev. 34:160-170.

Veinante P, Deschênes M. 2003. Single-cell study of motor cortex projections to the barrel field in rats. J Comp Neurol. 464:98-103.

White EL, DeAmicis RA. 1977. Afferent and efferent projections of the region in mouse sml cortex which contains the posteromedial barrel subfield. J Comp Neurol. 175:455-481.

Whitfield-Gabrieli S, Nieto-Castanon A. 2012. Conn: a functional connectivity toolbox for correlated and anticorrelated brain networks. Brain Connect. 2:125-141.

Xu N, Harnett MT, Williams SR, Huber D, O'Connor DH, Svoboda K, Magee JC. 2012. Nonlinear dendritic integration of sensory and motor input during an active sensing task. Nature. 492:247-251.

Zar JH. 1996. Biostatistical analysis. Prentice Hall, DeKalb, IL, United States

Zhang Z, Francisco EM, Holden JK, Dennis RG, Tommerdahl M. 2011. Somatosensory information processing in the aging population. Front Aging Neurosci. 3:18.

Zimmermann M, Verhagen L, de Lange FP, Toni I (2016) The Extrastriate body area computes desired goal states during action planning. eNeuro 3. https://www.ncbi.nlm.nih.gov/ pmc/articles/PMC4821904/ (Accessed February 7, 2019). 